\title{
Fibronectin in Fracture Healing: Biological Mechanisms and Regenerative Avenues
}

\author{
Jonathan Klavert and Bram C. J. van der Eerden* \\ Department of Internal Medicine, Erasmus University Medical Center, Rotterdam, Netherlands
}

The importance of extracellular matrix (ECM) proteins in mediating bone fracture repair is evident, and fibronectin (FN) has emerged as a pivotal regulator of this process. $\mathrm{FN}$ is an evolutionarily conserved glycoprotein found in all tissues of the body, and functions in several stages of fracture healing. FN acts as a three-dimensional scaffold immediately following trauma, guiding the assembly of additional ECM components. Furthermore, FN regulates cellular behavior via integrin-binding and growth factorbinding domains, promoting downstream responses including cell recruitment, proliferation and differentiation. Due to its diverse functions, the development of FNbased strategies to promote fracture healing is under intense research. In this review, we

OPEN ACCESS

Edited by:

Lorenzo Moroni,

Maastricht University, Netherlands

Reviewed by:

Aleksandra Leszczynska, University of California, San Diego, United States

Barbara Barboni,

University of Teramo, Italy

*Correspondence:

Bram C. J. van der Eerden b.vandereerden@erasmusmc.nl

Specialty section:

This article was submitted to Tissue Engineering and Regenerative Medicine,

a section of the journal Frontiers in Bioengineering and Biotechnology

Received: 02 February 2021 Accepted: 22 March 2021 Published: 16 April 2021

Citation:

Klavert $J$ and van der Eerden BCJ (2021) Fibronectin in Fracture Healing: Biological Mechanisms

and Regenerative Avenues. Front. Bioeng. Biotechnol. 9:663357. doi: 10.3389/fbioe.2021.663357 discuss the recent advancements in utilizing FN-based biomaterials, showing promise in tissue engineering and regenerative medicine applications.

Keywords: fibronectin, fracture healing, biomaterial, extracellular matrix, bone, regenerative medicine, tissue engineering, angiogenesis

\section{INTRODUCTION}

The skeletal system is involved in many roles, including mechanical load bearing and movement, soft tissue protection, and as a supportive niche for hematopoietic cells (Calvi et al., 2003). The performed functions of bone are dependent on its structure, thus resolving structural defects are crucial for maintaining homeostasis (reviewed in Florencio-Silva et al., 2015). Although bone is a highly dynamic tissue, large bone defects still represent a clinical challenge. Due to being one of the most common injuries worldwide (Amin et al., 2014), bone fractures cause high morbidity and economic burden to society. The elderly in particular are at an increased risk for developing fractures due to the high prevalence of osteoporosis in this demographic, a disease characterized by brittle bones estimated to affect $>14$ million people in the United States alone (National Osteoporosis Foundation, 2002).

Approximately $5-10 \%$ of bone fractures result in delayed healing or non-union, which may require surgical intervention (Einhorn, 1998). The currently accepted gold standard for treatment in such cases remains the use of bone graft, but are not without limitations (reviewed by Faour et al., 2011). Donor morbidity and limited tissue availability are concerns using autologous bone grafts, and while concern of sufficient tissue amounts can be bypassed with allogenic material, risk of disease transmission remains an issue (Delloye et al., 2007).

\section{BONE EXTRACELLULAR MATRIX}

These concerns and limitations highlight the need for developing novel strategies aimed at promoting bone regeneration, which can be achieved by further elucidating the molecular 
mechanisms involved in fracture healing. Of particular interest is the role of extracellular matrix (ECM), which comprises approximately $90 \%$ of bone tissue in mass (v/v) (Mansour et al., 2017). Additionally, the ECM is known to be a pivotal regulator of cellular behaviors including adhesion, migration, proliferation, and differentiation (Becerra-Bayona et al., 2012; Zeitouni et al., 2012). The significance of ECM function in bone regeneration has been demonstrated by the improved (clinical) performance of decellularized allogenic grafts, showing similar success to autogenic grafts (Al-Abedalla et al., 2015).

Many ECM molecules have been studied extensively throughout the years. This review seeks to integrate the findings related to one particular ECM protein, namely fibronectin (FN), which seems to be functionally relevant across several stages of fracture healing.

\section{STAGES OF FRACTURE HEALING}

Fracture healing is a tightly controlled process, which after the injury causing trauma can be divided into four main stages: (1) inflammation, (2) soft callus formation, (3) hard callus formation, and (4) bone remodeling. There seems to be temporal overlap between aforementioned stages of fracture healing (Schindeler et al., 2008), but making such distinctions is nevertheless practical to describe the general events that take place.

The initial stage of inflammation arises from damage to soft tissue surrounding the fracture, which leads to bleeding that later develops into a hematoma. Inflammation itself is tightly regulated as the inflammatory cascade is essential to initiate fracture healing, but must be appropriately resolved at later stages (for a recent review, see Maruyama et al., 2020). Pro-inflammatory cytokines secreted by resident macrophages promotes the recruitment of additional immune cells, including neutrophils and monocytes, in a positive feedback loop of acute inflammation (reviewed in Baht et al., 2018). Such an inflammatory microenvironment is crucial in recruiting mesenchymal stem cells (MSCs) to the site of injury via chemotaxis (i.e., interleukins [ILs] 1 and 6, TNF-alpha; Figure 1A). MSCs have the ability to differentiate into cells which regenerate skeletal elements (Buravkova and Anokhina, 2008; Schindeler et al., 2008; Watson et al., 2014), and this process is promoted by cellular crosstalk with immune cells (Chang et al., 2008; Raggatt et al., 2014; Vi et al., 2015). Conversely, MSCs have been described to possess an immunomodulatory role, mediated by release of soluble factors and cell-cell interactions, thus resolving the acute inflammatory response (reviewed in Munir et al., 2018).

Most fractures follow the endochondral ossification pathway, in which the final resulting bone is preceded by a soft callus composed of cartilage (Gerstenfeld et al., 2003). This soft callus serves to provide mechanical stability to the fracture, and acts as a rudimentary template for bone formation (Schindeler et al., 2008). To establish vasculature, endothelial cells (ECs) then invade the soft callous; a process in which MSCs play a crucial role, by producing angiogenic factors including vascular endothelial growth factor-A (VEGF-A) and platelet-derived growth factor-BB (PDGF-BB) known to enhance migration and proliferation of ECs (Chen et al., 2008). Recent studies have demonstrated that angiogenesis and osteogenesis are tightly coupled processes positively regulating each other, with intimate crosstalk occurring between specific EC subsets and bone progenitor cells (Kusumbe et al., 2014; Ramasamy et al., 2014).

Hard callous formation occurs in stable areas surrounding the soft callous, as the new bone forms in association with new blood vessels. Osteoblasts secrete the osteoid (primarily composed of type I collagen, osteopontin, and osteocalcin) into the ECM, which are then mineralized to form hydroxyapatite crystals ( $\mathrm{Li}$ et al., 2014). Remodeling of the mineralized (hard) callous is the final stage of fracture healing in which specialized cells-namely osteoclasts-resorb mineralized bone resulting in surface gaps then re-filled by osteoblasts (Schindeler et al., 2008).

\section{FIBRONECTIN STRUCTURE AND ASSEMBLY}

Fibronectin is an evolutionarily conserved glycoprotein, existing as a dimer composed of two nearly identical $\sim 250 \mathrm{kDa}$ subunits (Pankov and Yamada, 2002; Figure 2). FN is a ubiquitous component of ECM in all tissues, which regulates adhesion, growth, and differentiation of cells (Yamada and Clark, 1996). FN possesses key functions in dynamic tissue remodeling throughout embryonic development (Davidson et al., 2004; Larsen et al., 2006), and has been linked to disease progression in multiple contexts (Oyama et al., 1989; Inufusa et al., 1995; Wan et al., 2013).

Fibronectin protein can exist in 20 isoforms, arising from alternative splicing of a single pre-mRNA molecule (Kosmehl et al., 1996). Structurally, FN consists of types I, II, and III repeating units (FNI-FNIII) and a C-terminal dimerization site. FN domains include binding sites for ECM molecules (i.e., collagen, heparin, fibrin, and other $\mathrm{FN}$ molecules), and for cell-binding via integrin receptors (for a recent review on FN structure, see Bradshaw and Smith, 2014; Figure 2). FN is secreted in both a soluble form, termed plasma FN ( $\mathrm{pFN}$ ), and a less soluble form termed cellular $\mathrm{FN}$ (cFN). cFN protein structure is distinguished from $\mathrm{pFN}$ by the inclusive splicing of extra domain(s) A and/or B $(\mathrm{EDA}+/ \mathrm{EDB}+)$, promoted in part via SF2/ASF or SRp40 splicing enhancers, respectively (reviewed in White et al., 2008; Figure 2).

Fibronectin matrix structure is assembled into higher order fibrils of $>10 \mathrm{~nm}$ in diameter and tens of micrometers in length (Larsen et al., 2006). Three-dimensional assembly of FN is a highly dynamic cell dependent process, and this cellmatrix crosstalk is guided by key physical parameters (for a comprehensive review on FN assembly, see Singh et al., 2010 Annu Rev). Cell contractile forces are essential for FN matrix formation (Halliday and Tomasek, 1995), as cells tether FN-FN interactions. Conversely, contraction of the FN matrix exposes additional ("cryptic") binding sites for additional FN molecules (Aguirre et al., 1994; Hocking et al., 1994). 


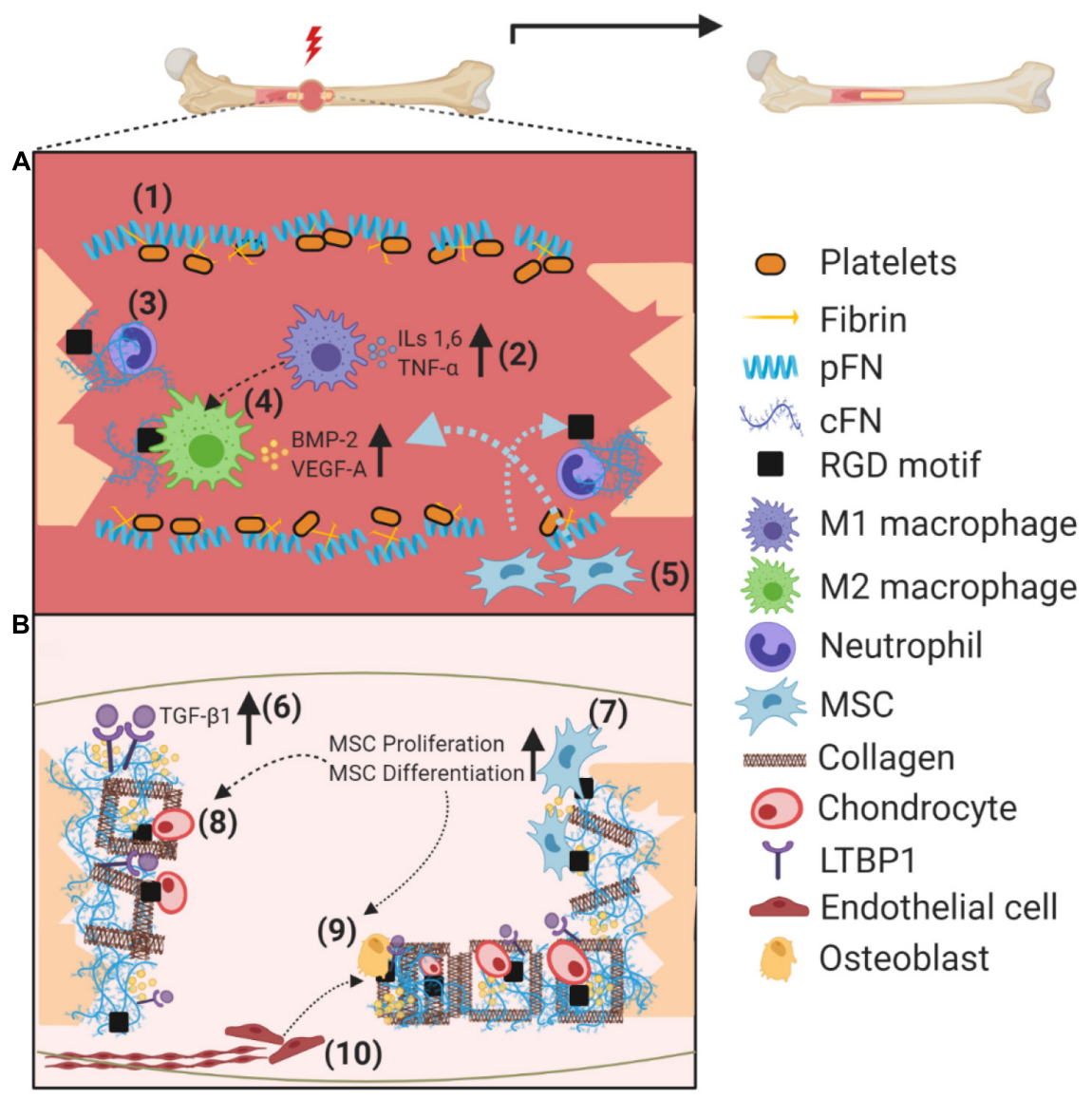

FIGURE 1 | Schematic illustration on the role of fibronectin (pFN and cFN) during fracture healing. (A) Soon after the fracture, pFN from the bloodstream in association with fibrin promotes clotting near the fracture site to form a hematoma (1). Acute inflammation is initiated by macrophages (M1), recruiting further immune cells via cytokine secretion (2). Neutrophils lay the groundwork for healing by secreting cFN as an "emergency ECM" (3). Cell binding to cFN RGD motifs switch macrophages to an anti-inflammatory (M2) phenotype, which secrete growth factors to recruit, among others, MSCs and ECs (4). Recruited MSCs infiltrate injury site and dock on integrin binding sites (5). (B) CFN orchestrates the deposition of additional proteins such as LTBP1 and collagen to the ECM, increasing availability of TGF- $\beta$ ligands, along with entrapment of additional growth factors such as BMP-2 (6). MSCs bound to cFN increase their proliferation rate via integrin signaling, followed by differentiation toward chondro/osteoprogenitors (7). Docking to cFN and growth factor regulation promotes cartilage formation via chondrocytes to stabilize the fracture (8). Attachment and proliferation of osteoblasts is promoted by integrin signaling, forming the hard callous (9), coupled to the recruitment/proliferation of endothelial cells and establishment of new vasculature also in part via integrin signaling (10). Abbreviations used: plasma fibronectin (pFN), cellular fibronectin (cFN), mesenchymal stem cell (MSC), latent TGF-binding protein 1 (LTBP1), transforming growth factor $\beta$ (TGF- $\beta$ ).

FN conformational alterations have also been demonstrated to affect cellular responses via integrin-binding specificity (Keselowsky et al., 2003, 2005) and growth factor (GF) availability (Wan et al., 2013).

\section{FIBRONECTIN IN FRACTURE HEALING}

Immediately following injury, $\mathrm{pFN}$ in the bloodstream promotes clotting near injured vessels, and seems to require fibrin association to enhance platelet aggregation (Wang et al., 2014). $\mathrm{pFN}$ has also been proposed to limit excessive clot formation distal from the injury site in vivo (Wang and $\mathrm{Ni}, 2016$ ). Although the described mechanism of pFN has not been studied in bone fracture models, it is reasonable to speculate that similar processes are involved during initial stages of blood vessel disruption and subsequent hematoma formation (Figure 1A). Unlike pFN, which is exclusively synthesized by hepatocytes, cFN is synthesized by several different cell types including fibroblasts, ECs, chondrocytes, and osteoblasts (Mao and Schwarzbauer, 2005). There is transcriptomic and immunohistochemical evidence that $\mathrm{cFN}$ is expressed, and dynamically regulated, in several stages of fracture healing (Liu et al., 2005; Kilian et al., 2008).

\section{Integration With ECM Through Integrins}

Cellular FN is assembled into a complex fibrillar matrix in a cell dependent process, and this cell-matrix interaction is largely mediated by integrins (Mao and Schwarzbauer, 2005). cFN binding to integrin receptors depend on a particular amino acid motif, consisting of Arginine-Glycine-Aspartate (RGD), which provide anchoring points for cells to the ECM. Activated integrin 


\section{Cellular FN}

(EDA+/EDB+)

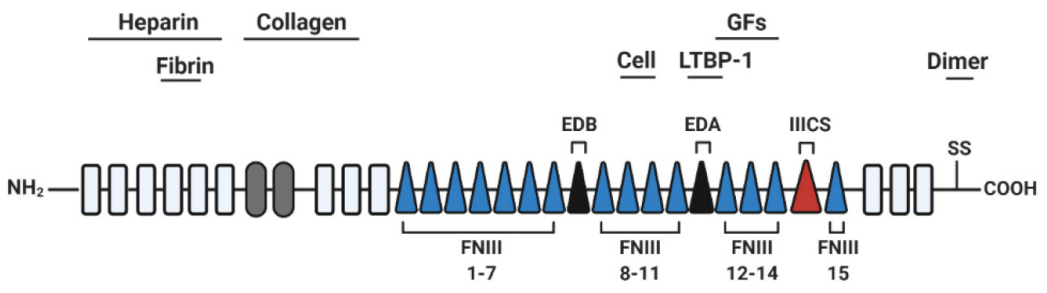

Plasma FN

(EDA-/EDB-)

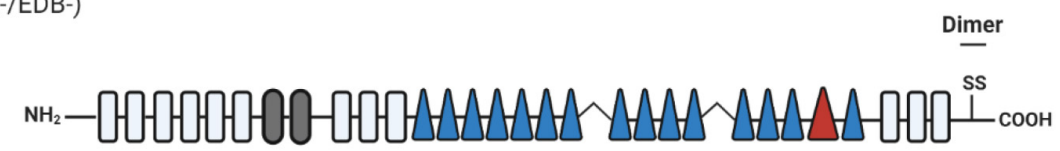

$\bigcap$ Type I (40AA)
$\bigcap_{\text {Type II (60AA) }}$
$\triangle$ Type III (90AA)

FIGURE 2 | Schematic illustration of fibronectin primary structure. Fibronectin (FN) is constitutively composed of evolutionarily conserved repeats: Type I (FNI; 12 units), Type II (FNII; 2 units), and Type III (FNIII; 15 units). Two FN monomers bind at the C-terminal dimerization site. Cellular FN isoforms are composed of alternatively spliced extra domains A/B (EDA/EDB), and variable portions of type III connecting segment (IIICS). Plasma FN lacks both EDA and EDB domains entirely. Known heparin, collagen, and fibrin-binding domains are depicted throughout FN, along with a promiscuous growth factor (GF)-binding domain (FNIII repeats 12-14). FNIII repeats 9-10 contain RGD motifs to bind integrins which mediate cell attachment and downstream signaling. Abbreviations used: growth factors (GFs), amino acids (AA), extra domain A/B (EDA/EDB), type III connecting segment (IIICS), arginine-glycine-aspartate (RGD).

receptors can, in part, direct cytoskeletal arrangements, which stretch the FN molecules and promote a positive feedback loop of fibril formation (Antia et al., 2008). Integrin $\alpha 5 ß 1$ expressed on osteogenic cells and fibroblasts, along with $\alpha \mathrm{V} \beta 3$ expressed on osteogenic cells and ECs, seem to play a crucial role in mediating cellular attachment to bone ECM (Parisi et al., 2019b).

In addition to regulating cell adhesion and fibril formation, FN-mediated integrin signaling can also alter cellular behavior itself. For instance, recombinant FN fragment containing the integrin-binding domain (FNIII 9-10) was shown to promote proliferation and differentiation of osteoblasts in vitro (Kim et al., 2003). Comparable performance of this FN fragment to native FN protein suggests that integrin signaling is a main factor in osteoblast proliferation and differentiation, thus positively contributing to fracture healing. The FNIII 9-10 integrin-binding domain was also found to enhance migration of both MSCs and ECs in vitro, providing further evidence of its importance in bone regeneration (Martino et al., 2011). FN-mediated integrin signaling can also alter cellular phenotypes, as has been observed for macrophages. Lv et al. (2018) have recently demonstrated that macrophages interact with $\mathrm{FN}$ via $ß 1$ integrins, converting (proinflammatory) M1 macrophages into an anti-inflammatory M2 phenotype in vitro (Figure 1A).

There is evidence that $\mathrm{cFN}$ is synthesized by inflammatory cells, neutrophils in particular, which infiltrate the fracture hematoma immediately within 48 h post-injury (Bastian et al., 2016). It is hypothesized that cFN acts as an "emergency ECM" at this timepoint, before stromal cells (i.e., MSCs and their progeny) infiltrate approximately 5 days post-injury and further modify the matrix (Bastian et al., 2016). This is consistent with the structure of $\mathrm{cFN}$, which contains binding domains for various ECM proteins such as collagens I and III, fibulin-1, fibrinogen, thrombospondin-1, and others (reviewed in Dallas et al., 2005). Such an "emergency ECM" composed of cFN could act as an initial scaffold for additional ECM molecules. Several studies have demonstrated that the three-dimensional cFN matrix plays an important role in orchestrating the spatiotemporal deposition of additional ECM molecules previously mentioned (Sottile and Hocking, 2002; Sottile et al., 2007).

\section{Interaction With Growth Factors}

Fibronectin has also been shown to bind a variety of GFs relevant to bone regeneration (Martino et al., 2011). A study conducted by Martino and Hubbell (2010) using recombinant FN fragments which strongly bind such GFs, significantly enhanced bone regeneration in rat models of critical-sized bone defect (Martino et al., 2011). In particular, the authors report that FN type III repeats 9-10 and 12-14 (FN III 9-10/12-14) promoted GF binding for chemotactic recruitment and proliferation of ECs and MSCs (i.e., VEGF-A, BMP-2, and PDGF-BB; Figure 1B; 
Martino et al., 2011). Binding of GFs to FN III 9-10/12-14 is unlikely to be the only mechanism involved, as functional synergy with integrins (namely $\alpha 5 ß 1$ and $\alpha \mathrm{V} \beta 3$ ) was evident.

Other crucial GFs in fracture healing are those of the transforming growth factor (TGF) family, which have been shown to recruit, among others, MSCs, fibroblasts and chondro/osteoprogenitors (Narine et al., 2006; Mendelson et al., 2011). There is a body of evidence supporting a non-redundant role of $\mathrm{cFN}$ in sequestering TGF ligands into the ECM. The cFN scaffold orchestrates deposition of latent TGF-binding protein 1 (LTBP-1), which seems to interact preferentially with the EDA+ domain of $\mathrm{cFN}$, resulting in subsequent TGF-beta ligand incorporation (Klingberg et al., 2018; Figure 1B, blue circles). In addition to its role in chemotactic recruitment, TGF- $\beta$ has been shown to promote callous formation via chondrocyte and osteoblast proliferation (Li et al., 2005), and differentiation (Bonewald and Dallas, 1994; Zhang et al., 2021). Interestingly, TGF- $\beta$ administration also strongly upregulates VEGF mRNA in osteoblasts in vitro, which could promote osteogenesis via regulating the coupled process of angiogenesis (Saadeh et al., 1999). pFN is also know to bind GFs, as it contains the main GF-binding domains (FN III 12-14) (Prasad and Clark, 2018). Coupled with the observations that pFN from the circulation contributes to local tissue ECM, its potential role in promoting bone healing via GF binding cannot be ruled out (Moretti et al., 2007).

\section{RECENT ADVANCEMENTS IN FIBRONECTIN-BASED BIOMATERIALS}

Due to its diverse properties in regulating both osteogenic and supporting cell types, along with orchestrating the assembly of various other ECM proteins and GFs, incorporating FN into regenerative medicine applications is under intensive research (Xing et al., 2017; Cheng et al., 2018; Escoda-Francolí et al., 2018; Guillem-Marti et al., 2018; Parisi et al., 2019a; Sánchez-Garcés et al., 2020; Toffoli et al., 2020; Trujillo et al., 2020; Zhang et al., 2020).

Lee et al. (2019) recently developed an FN fusion protein, containing FNIII 9-10 domain and elastin like peptides (FNELP) to more reliably recapitulate mechanical properties of native ECM. FN-ELP significantly enhanced MSC adhesion, proliferation, and osteogenic differentiation in vitro. This approach could thus provide improved ex vivo expansion and differentiation of MSCs into desired cell type(s) for stem cellbased therapies. Another noteworthy finding demonstrated that the GF binding domain (FNIII 12-14) could also facilitate cell attachment and spreading to titanium surface when an RGD gain-of-function mutation was introduced within (Guillem-Marti et al., 2019).

In addition to $\mathrm{FN}$ fragments which can be immobilized, the development of hydrogels also seems to be a promising avenue. Such hydrogels can potentially be administered surrounding the fracture site directly, which could alleviate the issue of difficult-toaccess areas when using grafts. Ao et al. (2020) recently developed a hydrogel composed of FN, fibrin glue, and heparin. In addition to reliably mimicking native ECM, this hydrogel could sustain the slow release of BMP-2-circumventing an issue persistent in previous carriers of BMP-2 administration. Their hydrogel managed to efficiently induce osteoblastic differentiation of MC3T3-E1 cells in vitro, and significantly promote fracture healing in vivo (Ao et al., 2020). Another promising biomaterial seems to be the use of FN in combination with nanotubulesmimicking collagen fibers-which is both injectable and can selfassemble (Zhou et al., 2020). The authors report significantly improved human MSC adhesion and migration into the "NanoMatrix" in vitro (Zhou et al., 2020).

\section{FUTURE OUTLOOK}

Despite the progress made, several biological questions remain unanswered. The negative relationship between aging and fracture healing has been well-described, and aberrations in FN functionality could mediate this reduced healing capacity. Interestingly, it has been shown that there is a significant effect of age on alternative splicing of $\mathrm{FN}$ both in vivo and in vitro (Magnuson et al., 1991), but the functional consequences and whether it is biologically significant in fracture healing, is still unknown. A connection between agerelated FN (alternative) splicing and TGF- $\beta$ signaling could potentially be made. As LTBP1 preferentially interacts with EDA+ FN (Klingberg et al., 2018), and levels of EDA+ FN are subject to age related changes, the deposition of LTBP1 could likely be affected.

Interestingly, relatively little is known about the role of FN during bone remodeling. There have been some demonstrations which suggest FN can upregulate osteoclast activity in vitro (Gramoun et al., 2010), but studying the complete mechanisms underlying this could prove fruitful for our knowledge on bone remodeling. Furthermore, although studies have conclusively demonstrated the importance of immune cell crosstalk in promoting bone regeneration (Chang et al., 2008; Raggatt et al., 2014; Vi et al., 2015), it remains unexplored in the context of FN-mediated fracture healing.

\section{CONCLUDING REMARKS}

Bone fractures will become increasingly common in the aging population, causing significant morbidity and financial burden. The involvement of ECM in fracture healing has been welldescribed along with its key molecular players, but recent evidence is further accumulating for the crucial role of FN. As it plays an important role in various stages of the healing cascade, studying FN functionality further, along with its incorporation into treatment strategies, could be of great utility.

Much attention has been devoted to developing FN-based biomaterials, and such three-dimensional scaffolds-especially those which can exert temporal control on GF availabilityseem highly promising. Further in vivo studies are warranted to evaluate their efficacy, perhaps investigating not just their effect on osteoprogenitors and MSCs, but to the highly relevant ECs and immune cell subsets as well. Several of the research 
lines summarized in this review have also highlighted the functional importance in utilizing recombinant FN fragments. Such recombinant fragments could pave the way for upscaling this approach, making it more feasible to translate into treatment strategies.

\section{REFERENCES}

Aguirre, K. M., McCormick, R. J., and Schwarzbauer, J. E. (1994). Fibronectin self-association is mediated by complementary sites within the amino-terminal one-third of the molecule. J. Biol. Chem. 269, 27863-27868. doi: 10.1016/s00219258(18)46866-4

Al-Abedalla, K., Torres, J., Cortes, A. R., Wu, X., Nader, S. A., Daniel, N., et al. (2015). boneaugmented with allograft onlays for implant placement could be comparable withnative bone. J. Oral Maxillofac Surg. 73, 2108-2122. doi: 10. 1016/j.joms.2015.06.151

Amin, S., Achenbach, S. J., Atkinson, E. J., Khosla, S., and Melton, L. J. III (2014). Trends in fracture incidence: a population-based study over 20 years. J. Bone Mineral Res. 29, 581-589. doi: 10.1002/jbmr.2072

Antia, M., Baneyx, G., Kubow, K. E., and Vogel, V. (2008). Fibronectin in aging extracellular matrixfibrils is progressively unfolded by cells and elicits an enhanced rigidityresponse. Faraday Discuss 139, 229-49; discussion309$25,419-20$.

Ao, Q., Wang, S., He, Q., Ten, H., Oyama, K., Ito, A., et al. (2020). Fibrin glue/fibronectin/heparin-based delivery system of BMP2 inducesosteogenesis in MC3T3-E1 cells and bone formation in rat calvarial critical-sizeddefects. ACS Appl. Mater. Interfaces 12, 13400-13410. doi: 10.1021/acsami.0c01371

Baht, G. S., Vi, L., and Alman, B. A. (2018). The role of the immune cells in fracture healing. Curr. Osteoporos. Rep. 16, 138-145. doi: 10.1007/s11914-018-0423-2

Bastian, O. W., Koenderman, L., Alblas, J., Leenen, L. P., and Blokhuis, T. J. (2016). Neutrophils contribute to fracture healing by synthesizing fibronectin+ extracellular matrix rapidly after injury. Clin. Immunol. 164, 78-84. doi: 10. 1016/j.clim.2016.02.001

Becerra-Bayona, S., Guiza-Arguello, V., Qu, X., Munoz-Pinto, D. J., and Hahn, M. S. (2012). Influence ofselect extracellular matrix proteins on mesenchymal stem cell osteogeniccommitment in three-dimensional contexts. Acta Biomater. 8, 4397-4404. doi: 10.1016/j.actbio.2012.07.048

Bonewald, L. F., and Dallas, S. L. (1994). Role of active and latent transforming growth factor $\beta$ in bone formation. J. Cell. Biochem. 55, 350-357. doi: 10.1002/ jcb. 240550312

Bradshaw, M. J., and Smith, M. L. (2014). Multiscale relationships between fibronectin structure and functional properties. Acta Biomater. 10, 1524-1531. doi: 10.1016/j.actbio.2013.08.027

Buravkova, L. B., and Anokhina, E. B. (2008). [Mesenchymal stromal progenitor cells: generalcharacteristics and functional state in low oxygen tension]. Ross. Fiziol. Zh. Im. I M Sechenova 94, 737-757.

Calvi, L. M., Adams, G. B., Weibrecht, K. W., Weber, J. M., Olson, D. P., Knight, M. C., et al. (2003). Osteoblastic cells regulate the haematopoietic stem cell niche. Nature 425, 841-846. doi: 10.1038/nature02040

Chang, M. K., Raggatt, L. J., Alexander, K. A., Kuliwaba, J. S., Fazzalari, N. L., Schroder, K., et al. (2008). Osteal tissue macrophages are intercalated throughout human and mouse bone lining tissues and regulate osteoblast function in vitro and in vivo. J. Immunol. 181, 1232-1244. doi: 10.4049/ jimmunol.181.2.1232

Chen, L., Tredget, E. E., Wu, P. Y., and Wu, Y. (2008). Paracrine factors of mesenchymal stem cells recruit macrophages and endothelial lineage cells and enhance wound healing. PLoS One 3:e1886. doi: 10.1371/journal.pone.0001886

Cheng, Z. A., Alba-Perez, A., Gonzalez-Garcia, C., Donnelly, H., LlopisHernandez, V., Jayawarna, V., et al. (2018). Nanoscale coatingsfor ultralow dose BMP-2-driven regeneration of critical-sized bone defects. Adv. Sci. (Weinh) 6:1800361. doi: 10.1002/advs.201800361

Dallas, S. L., Sivakumar, P., Jones, C. J., Chen, Q., Peters, D. M., Mosher, D. F., et al. (2005). Fibronectin regulates latent transforming growth factor-beta (TGFbeta) by controlling matrix assembly of latent TGF betabinding protein-1. J. BiolChem. 280, 18871-18880. doi: $10.1074 /$ jbc.m4107 62200

\section{AUTHOR CONTRIBUTIONS}

JK performed the literature research, wrote the manuscript, and designed the figure. BE revised the manuscript. Both authors contributed to the article and approved the submitted version.

Davidson, L. A., Keller, R., and DeSimone, D. W. (2004). Assembly and remodeling of the fibrillar fibronectin extracellular matrix during gastrulation and neurulation in Xenopus laevis. Dev. Dyn. 231, 888-895. doi: 10.1002/dvdy. 20217

Delloye, C., Cornu, O., Druez, V., and Barbier, O. (2007). Bone allografts: what they can offer and what they cannot. J. Bone Joint Surg. Br. 89, 574-579. doi: 10.1302/0301-620x.89b5.19039

Einhorn, T. A. (1998). The cell and molecular biology of fracture healing. Clin. Orthop. Relat. Res. (355 Suppl), S7-S21.

Escoda-Francolí, J., Sánchez-Garcés, M. Á, Gimeno-Sandig, Á, Muñoz-Guzón, F., Barbany-Cairó, J. R., Badiella-Busquets, L., et al. (2018). Guided bone regeneration using beta-tricalcium phosphate with and without fibronectinAn experimental study in rats. Clin. Oral Implants Res. 29, 1038-1049. doi: $10.1111 /$ clr. 13370

Faour, O., Dimitriou, R., Cousins, C. A., and Giannoudis, P. V. (2011). The use of bone graftsubstitutes in large cancellous voids: any specific needs? Injury 42(Suppl. 2), S87-S90. doi: 10.1016/j.injury.2011.06.020

Florencio-Silva, R., Sasso, G. R., Sasso-Cerri, E., Simões, M. J., and Cerri, P. S. (2015). Biology of bone tissue: structure, function, and factors that influence bone cells. BioMed. Res. Int. 2015:421746.

Gerstenfeld, L. C., Cho, T. J., Kon, T., Aizawa, T., Tsay, A., Fitch, J., et al. (2003). Impaired fracture healing in the absence of TNF- $\alpha$ signaling: The role of TNF$\alpha$ in endochondral cartilage resorption. J. Bone Mineral Res. 18, 1584-1592. doi: 10.1359/jbmr.2003.18.9.1584

Gramoun, A., Azizi, N., Sodek, J., Heersche, J. N., Nakchbandi, I., and Manolson, M. F. (2010). Fibronectin inhibits osteoclastogenesis while enhancing osteoclast activity via nitric oxide and interleukin- $1 \beta$-mediated signaling pathways. J. Cell Biochem. 111, 1020-1034. doi: 10.1002/jcb.22791

Guillem-Marti, J., Boix-Lemonche, G., Gugutkov, D., Ginebra, M. P., Altankov, G., and Manero, J. M. (2018). Recombinant fibronectin fragment III810 /polylactic acid hybrid nanofibers enhance the bioactivity of titanium surface. Nanomedicine 13, 899-912. doi: 10.2217/nnm-2017-0342

Guillem-Marti, J., Gelabert, M., Heras-Parets, A., Pegueroles, M., Ginebra, M. P., and Manero, J. M. R. G. D. (2019). Mutation of the Heparin Binding II Fragment of Fibronectin for GuidingMesenchymal Stem Cell Behavior on Titanium Surfaces. ACS Appl. Mater. Interfaces 11, 3666-3678. doi: 10.1021/ acsami.8b17138

Halliday, N. L., and Tomasek, J. J. (1995). Mechanical properties of the extracellular matrix influence fibronectin fibril assembly in vitro. Exp. Cell Res. 217, 109-117. doi: 10.1006/excr.1995.1069

Hocking, D. C., Sottile, J., and McKeown-Longo, P. J. (1994). Fibronectin's III1 module contains a conformation-dependent binding site for the aminoterminal region of fibronectin. J. Biol. Chem. 269, 19183-19191. doi: 10.1016/ s0021-9258(17)32292-5

Inufusa, H., Nakamura, M., Adachi, T., Nakatani, Y., Shindo, K., Yasutomi, M., et al. (1995). Localization of oncofetal and normal fibronectin in colorectal cancer. Correlation with histologic grade, liver metastasis, and prognosis. Cancer 75, 2802-2808. doi: 10.1002/1097-0142(19950615)75:12<2802::aidcncr2820751204>3.0.co;2-o

Keselowsky, B. G., Collard, D. M., and García, A. J. (2003). Surface chemistry modulates fibronectin conformation and directs integrin binding and specificity to control cell adhesion. J. Biomed. Mater. Res. Part A. 66, 247-259. doi: 10.1002/jbm.a.10537

Keselowsky, B. G., Collard, D. M., and García, A. J. (2005). Integrin binding specificity regulates biomaterial surface chemistry effects on cell differentiation. Proc. Natl. Acad. Sci.U.S.A. 102, 5953-5957. doi: 10.1073/pnas.0407356102

Kilian, O., Dahse, R., Alt, V., Zardi, L., Hentschel, J., Schnettler, R., et al. (2008). mRNA expression and protein distribution of fibronectin splice variants and high-molecular weight tenascin- $\mathrm{C}$ in different phases of human fracture healing. Calcif. Tissue Int. 83, 101-111. doi: 10.1007/s00223-008-9156-z 
Kim, T. I., Jang, J. H., Chung, C. P., and Ku, Y. (2003). Fibronectin fragment promotes osteoblast-associated gene expression and biological activity of human osteoblast-like cell. Biotechnol. Lett. 25, 2007-2011. doi: 10.1023/b: bile.0000004393.02839.d8

Klingberg, F., Chau, G., Walraven, M., Boo, S., Koehler, A., Chow, M. L., et al. (2018). The fibronectin ED-A domain enhancesrecruitment of latent TGF$\beta$-binding protein-1 to the fibroblast matrix. J. Cell Sci. 131, jcs201293. doi: $10.1242 /$ jcs. 201293

Kosmehl, H., Berndt, A., and Katenkamp, D. (1996). Molecular variants of fibronectin and laminin: structure, physiological occurrence and histopathological aspects. Virchows Arch. 429, 311-322.

Kusumbe, A. P., Ramasamy, S. K., and Adams, R. H. (2014). Coupling of angiogenesis and osteogenesis by a specific vessel subtype in bone. Nature 507, 323-328. doi: 10.1038/nature13145

Larsen, M., Wei, C., and Yamada, K. M. (2006). Cell and fibronectin dynamics during branching morphogenesis. J. Cell Sci. 119, 3376-3384. doi: 10.1242/jcs. 03079

Lee, S., Kim, J. E., Seo, H. J., and Jang, J. H. (2019). Design of fibronectin type III domains fused toan elastin-like polypeptide for the osteogenic differentiation of humanmesenchymal stem cells. Acta Biochim. Biophys. Sin. (Shanghai). 51, 856-863. doi: 10.1093/abbs/gmz063

Li, J., Baker, B. A., Mou, X., Ren, N., Qiu, J., Boughton, R. I., et al. (2014). Biopolymer/Calcium phosphate scaffolds for bone tissue engineering. $A d v$. Healthc. Mater. 3, 469-484. doi: 10.1002/adhm.201300562

Li, T. F., O'Keefe, R. J., and Chen, D. (2005). TGF- $\beta$ signaling in chondrocytes. Front. Biosci. 10:681. doi: 10.2741/1563

Liu, S. H., Cheng, G., Li, S. W., Tian, W. D., and Liu, L. (2005). [Study on mRNA expression of fibronectin and integrin beta1 during fracture healing]. Hua Xi Kou Qiang Yi Xue Za Zhi. 23, 434-437.

Lv, L., Xie, Y., Li, K., Hu, T., Lu, X., Cao, Y., et al. (2018). Unveiling the mechanism of surfacehydrophilicity-modulated macrophage polarization. Adv. Healthc. Mater. 7, e1800675. doi: 10.1002/adhm.201800675

Magnuson, V. L., Young, M., Schattenberg, D. G., Mancini, M. A., Chen, D. L., and Steffensen, B. (1991). KlebeRJ. The alternative splicing of fibronectin premRNA is altered during aging and in response to growth factors. J. Biol. Chem. 266, 14654-14662. doi: 10.1016/s0021-9258(18)98735-1

Mansour, A., Mezour, M. A., Badran, Z., and Tamimi, F. (2017). (*) Extracellular matrices for boneregeneration: a literature review. Tissue Eng. Part A. 23, 1436-1451. doi: 10.1089/ten.TEA.2017.0026

Mao, Y., and Schwarzbauer, J. E. (2005). Fibronectin fibrillogenesis, a cell-mediated matrix assembly process. Matrix Biol. 24, 389-399. doi: 10.1016/j.matbio.2005. 06.008

Martino, M. M., Hubbell, J. A. (2010). The 12th-14th type III repeats of fibronectin function asa highly promiscuous growth factor-binding domain. FASEB J. 24, 4711-4721. doi: 10.1096/fj.09-151282

Martino, M. M., Tortelli, F., Mochizuki, M., Traub, S., Ben-David, D., Kuhn, G. A., et al. (2011). Engineering the growth factor microenvironment with fibronectin domains to promote wound and bone tissue healing. Sci. Transl. Med. 3:100ra89. doi: 10.1126/scitranslmed.3002614

Maruyama, M., Rhee, C., Utsunomiya, T., Zhang, N., Ueno, M., Yao, Z., et al. (2020). Modulation of the inflammatory response and bone healing. Front. Endocrinol. 11:386. doi: 10.3389/fendo.2020.00386

Mendelson, A., Frank, E., Allred, C., Jones, E., Chen, M., Zhao, W., et al. (2011). Chondrogenesisby chemotactic homing of synovium, bone marrow, and adipose stem cells in vitro. FASEB J. 25, 3496-3504. doi: 10.1096/fj.10176305

Moretti, F. A., Chauhan, A. K., Iaconcig, A., Porro, F., Baralle, F. E., and Muro, A. F. (2007). A major fraction of fibronectin present in the extracellular matrix of tissues is plasma-derived. J. Biol. Chem. 282, 28057-28062. doi: 10.1074/jbc. m611315200

Munir, H., Ward, L. S. C., and McGettrick, H. M. (2018). Mesenchymal stem cells as endogenous regulatorsof inflammation. Adv. Exp. Med. Biol. 1060, 73-98. doi: 10.1007/978-3-319-78127-3_5

Narine, K., De Wever, O., Van Valckenborgh, D., Francois, K., Bracke, M., DeSmet, S., et al. (2006). Growth factor modulation of fibroblast proliferation,differentiation, and invasion: implications for tissue valve engineering. Tissue Eng. 12, 2707-2716. doi: 10.1089/ten.2006.12. 2707
National Osteoporosis Foundation (2002). America's Bone Health: The State of Osteoporosis and low Bone Mass in Our nation. Washington DC: National Osteoporosis Foundation.

Oyama, F., Hirohashi, S., Shimosato, Y., Titani, K., and Sekiguchi, K. (1989). Deregulation of alternative splicing of fibronectin pre-mRNA in malignant human liver tumors. J. Biol. Chem. 264, 10331-10334. doi: 10.1016/s00219258(18)81621-0

Pankov, R., and Yamada, K. M. (2002). Fibronectin at a glance. J. Cell Sci. 115(Pt20), 3861-3863. doi: $10.1242 /$ jcs.00059

Parisi, L., Toffoli, A., Bianchi, M. G., Bergonzi, C., Bianchera, A., Bettini, R., et al. (2019a). Functional fibronectin adsorption on aptamer-doped chitosanmodulates cell morphology by integrin-mediated pathway. Materials (Basel) 12, E812. doi: 10.3390/ma12050812

Parisi, L., Toffoli, A., Ghezzi, B., Mozzoni, B., Lumetti, S., and Macaluso, G. M. (2019b). A glance on the role of fibronectin in controlling cell response at biomaterialinterface. Jpn Dent. Sci. Rev. 56, 50-55. doi: 10.1016/j.jdsr.2019.11. 002

Prasad, A., and Clark, R. A. (2018). Fibronectin interaction with growth factors in the context of general ways extracellular matrix molecules regulate growth factor signaling. G. Ital. Dermatol. Venereol. 153, 361-374.

Raggatt, L. J., Wullschleger, M. E., Alexander, K. A., Wu, A. C., Millard, S. M., Kaur, S., et al. (2014). Fracture healing via periosteal callus formation requires macrophages for both initiation and progression of early endochondral ossification. Am. J. Pathol. 184, 3192-3204. doi: 10.1016/j.ajpath.2014.08.017

Ramasamy, S. K., Kusumbe, A. P., Wang, L., and Adams, R. H. (2014). Endothelial notch activity promotesangiogenesis and osteogenesis in bone. Nature 507, 376-380. doi: 10.1038/nature13146

Saadeh, P. B., Mehrara, B. J., Steinbrech, D. S., Dudziak, M. E., Greenwald, J. A., Luchs, J. S., et al. (1999). Transforming growth factor-betal modulates the expression of vascular endothelial growth factor by osteoblasts. Am. J. Physiol. 277, C628-C637. doi: 10.1152/ajpcell.1999.277.4.C628

Sánchez-Garcés, M. Á, Camps-Font, O., Escoda-Francolí, J., Muñoz-Guzón, F., Toledano-Serrabona, J., and Gay-Escoda, C. (2020). Short time guided bone regeneration using beta-tricalcium phosphate with and without fibronectinAn experimental study in rats. Med. Oral Patol. Oral. Cir. Bucal 25:e532. doi: $10.4317 /$ medoral.23564

Schindeler, A., McDonald, M. M., Bokko, P., and Little, D. G. (2008). Bone remodeling during fracturerepair: the cellular picture. Semin. Cell Dev. Biol. 19, 459-466. doi: 10.1016/j.semcdb.2008.07.004

Singh, P., Carraher, C., and Schwarzbauer, J. E. (2010). Assembly of fibronectin extracellular matrix. Ann. Rev. Cell Dev. Biol. 26, 397-419.

Sottile, J., and Hocking, D. C. (2002). Fibronectin polymerization regulates the composition andstability of extracellular matrix fibrils and cell-matrix adhesions. Mol. BiolCell. 13, 3546-3559. doi: 10.1091/mbc.e02-01-0048

Sottile, J., Shi, F., Rublyevska, I., Chiang, H. Y., Lust, J., and Chandler, J. (2007). Fibronectin-dependent collagen I deposition modulates the cell response to fibronectin. Am. J. Physiol. Cell Physiol. 293, C1934-C1946.

Toffoli, A., Parisi, L., Bianchi, M. G., Lumetti, S., Bussolati, O., and Macaluso, G. M. (2020). Thermaltreatment to increase titanium wettability induces selective proteins adsorption from blood serum thus affecting osteoblasts adhesion. Mater. Sci Eng.. C Mater. Biol. Appl. 107:110250. doi: 10.1016/j.msec.2019. 110250

Trujillo, S., Gonzalez-Garcia, C., Rico, P., Reid, A., Windmill, J., Dalby, M. J., et al. (2020). Engineered 3D hydrogels with full-length fibronectin that sequester and present growth factors. Biomaterials 252:120104. doi: 10.1016/j.biomaterials. 2020.120104

Vi, L., Baht, G. S., Whetstone, H., Ng, A., Wei, Q., Poon, R., et al. (2015). Macrophages promote osteoblastic differentiation in-vivo: implications in fracture repair and bone homeostasis. J. Bone Mineral Res. 30, 1090-1102. doi: $10.1002 /$ jbmr.2422

Wan, A. M., Chandler, E. M., Madhavan, M., Infanger, D. W., Ober, C. K., Gourdon, D., et al. (2013). Fibronectin conformation regulates the proangiogenic capability of tumor-associated adipogenic stromal cells. Biochim. Biophy. Acta 1830, 4314-4320. doi: 10.1016/j.bbagen.2013. 03.033

Wang, Y., and Ni, H. (2016). Fibronectin maintains the balance between hemostasis and thrombosis. Cell. Mol. Life Sci. 73, 3265-3277. doi: 10.1007/ s00018-016-2225-y 
Wang, Y., Reheman, A., Spring, C. M., Kalantari, J., Marshall, A. H., Wolberg, A. S., et al. (2014). Plasma fibronectin supports hemostasis and regulates thrombosis. J. Clin. Invest. 124, 4281-4293. doi: $10.1172 /$ jci74630

Watson, L., Elliman, S. J., and Coleman, C. M. (2014). From isolation to implantation: a concisereview of mesenchymal stem cell therapy in bone fracture repair. Stem Cell Res. Ther. 5:51. doi: 10.1186/scrt 439

White, E. S., Baralle, F. E., and Muro, A. F. (2008). New insights into form and function of fibronectin splice variants. J. Pathol. 216, 1-14. doi: 10.1002/path. 2388

Xing, J., Mei, T., Luo, K., Li, Z., Yang, A., Li, Z., et al. (2017). A nano-scaled and multi-layered recombinant fibronectin/cadherin chimera composite selectively concentrates osteogenesis-related cells and factors to aid bone repair. Acta biomaterialia 53, 470-482. doi: 10.1016/j.actbio.2017.02.016

Yamada, K. M., and Clark, R. A. F. (1996). The Molecular and Cellular Biology of Wound Repair. New York NY: Springer,.

Zeitouni, S., Krause, U., Clough, B. H., Halderman, H., Falster, A., Blalock, D. T., et al. (2012). Human mesenchymal stem cell-derived matrices for enhancedosteoregeneration. Sci. Transl. Med. 4:132ra55.

Zhang, H., Chen, X., Xue, P., Ma, X., Li, J., and Zhang, J. (2020). FN1 promotes chondrocyte differentiation and collagen production via
TGF- $\beta /$ PI3K/Akt pathway in mice with femoral fracture. Gene 769: 145253.

Zhang, H., Chen, X., Xue, P., Ma, X., Li, J., and Zhang, J. (2021). FN1 promotes chondrocyte differentiation and collagen production via TGF- $\beta / \mathrm{PI} 3 \mathrm{~K} / \mathrm{Akt}$ pathway in mice with femoral fracture. Gene 769:145253. doi: 10.1016/j.gene. 2020.145253

Zhou, L., Yau, A., Yu, H., Kuhn, L., Guo, W., and Chen, Y. (2020). Selfassembled biomimetic Nano-Matrixfor stem cell anchorage. J. Biomed Mater. Res. A. 108, 984-991. doi: 10.1002/jbm.a.36875 doi: 10.1002/jbm.a.3 6875

Conflict of Interest: The authors declare that the research was conducted in the absence of any commercial or financial relationships that could be construed as a potential conflict of interest.

Copyright (c) 2021 Klavert and van der Eerden. This is an open-access article distributed under the terms of the Creative Commons Attribution License (CC BY). The use, distribution or reproduction in other forums is permitted, provided the original author(s) and the copyright owner(s) are credited and that the original publication in this journal is cited, in accordance with accepted academic practice. No use, distribution or reproduction is permitted which does not comply with these terms. 